\title{
Time-efficient Significance Measure for Discovering Spatiotemporal Co-occurrences from Data with Unbalanced Characteristics
}

\author{
Berkay Aydin, Vijay Akkineni, Rafal Angryk \\ Department of Computer Science \\ Georgia State University \\ Atlanta, GA 30303, USA \\ \{baydin2, vakkineni1, rangryk\}@cs.gsu.edu
}

\begin{abstract}
Mining spatiotemporal co-occurrence patterns requires assessing the strength of co-occurrences among the instances of different feature types. Currently, a spatiotemporal version of the Jaccard measure is used for measuring the strength of spatiotemporal co-occurrences. We present an extended spatiotemporal version of the Jaccard measure $\left(J^{*}\right)$ that is more relevant and efficient for the task of STCOP mining. We also demonstrate the space and time efficiency of the $J^{*}$ with experimental evaluation.
\end{abstract}

\section{Categories and Subject Descriptors}

H.2.8 [Database Applications]: Data Mining-Spatial Data Mining and Knowledge Discovery

\section{Keywords}

Spatiotemporal co-occurrence pattern; interestingness measure; spatiotemporal knowledge discovery

\section{INTRODUCTION}

An important aspect of data mining research is the determination of the interestingness of patterns. Objective measures, such as support, confidence, correlation, and entropy, have been extensively used in frequent pattern mining $[6,9]$. There has been extensive research on understanding and assessing the quality, interestingness, and appropriateness of objective measures for different tasks and domains; however, there is no prevalent agreement on this issue, because the quality of the measures depends on the data mining task and domain [3]. Selecting the right measure is of great importance, as many measures create conflicting information due to their significantly different properties [10].

We propose a new significance measure for assessing the strength of a spatiotemporal co-occurrence in the context of spatiotemporal co-occurrence pattern (STCOP) mining

Permission to make digital or hard copies of all or part of this work for personal or classroom use is granted without fee provided that copies are not made or distributed for profit or commercial advantage and that copies bear this notice and the full citation on the first page. Copyrights for components of this work owned by others than ACM must be honored. Abstracting with credit is permitted. To copy otherwise, or republish, to post on servers or to redistribute to lists, requires prior specific permission and/or a fee. Request permissions from Permissions@ acm.org.

SIGSPATIAL'15 November 03-06, 2015, Bellevue, WA, USA

(C) 2015 ACM. ISBN 978-1-4503-3967-4/15/11 . \$ $\$ 15.00$

DOI: http://dx.doi.org/10.1145/2820783.2820871 from large datasets with unbalanced spatiotemporal characteristics [8]. STCOPs represent the feature types whose instances frequently co-occur in both space and time. Spatiotemporal co-occurrences are the events of spatiotemporal overlap among the instances of different feature types. Current algorithms use a spatiotemporal version of Jaccard $(J)$ measure for determining the strength of co-occurrences $[1,8]$. The instances are modeled as three dimensional objects, and the $J$ measure for co-occurrences is the ratio of intersection to union volume of the instances. However, using the $J$ measure leads to unfair assessments in certain cases. In this work, we introduce the $J^{*}$ measure to alleviate these issues, and increase the efficiency of the STCOP mining algorithms. Our contributions include: developing a novel and efficient objective measure, providing theoretical analysis on the antimonotonicity property of $J^{*}$, and verifying the applicability of the measure by providing experimental results showing the computational efficiency of $J^{*}$.

\subsection{Related Work and Motivation}

In spatiotemporal pattern mining, spatiotemporal relationships among objects are not explicit; thus, it is necessary to transform the implicit information to a transaction-like embodiment. Examples of such transformations in spatial and spatiotemporal pattern mining literature include spacedriven partitioning [7], event-centric [5], buffer-based [12], and distance-based neighborhooding [2] approaches.

In STCOP mining, a prevalent STCOP is a set of features, whose instances frequently and strongly co-occur together in both space and time. The prevalency of STCOPs is assessed by the participation index at the feature level [5]. Instances are considered to form a spatiotemporal co-occurrence if there exists a spatiotemporal overlap among instances [8]. This approach is similar to the buffer based model [12], where the buffers of spatial objects are tested with the spatial overlap predicate. In addition to spatiotemporal overlap predicate, the strength of a co-occurrence is measured by $J$. The strength of spatiotemporal co-occurrences is measured at the instance level, and implies the significance of a cooccurrence event.

STCOP mining algorithms are Apriori-based, and the cooccurrences are considered significant if they can pass a given co-occurrence coefficient (cce) threshold. Similarly, STCOPs are considered prevalent if they can pass a prevalence index $(p i)$ threshold. STCOP mining algorithms are dependent on the antimonotonic property of the prevalency 
and significance measures ${ }^{1}$.

Measuring the strength of spatiotemporal co-occurrences with $J$ leads to unfair assessments when the participating instances have significantly different spatiotemporal characteristics (such as lifespan, area, volume). When calculating the strength, $J$ measure considers that all parts of instance trajectories equally contribute to the strength. However, when participating instances have unbalanced characteristics, it is necessary to consider only relevant segments of the instances. For example, the co-occurrence of a large, longlasting instance and a small, short-lived instance leads to a small $J$ value, even when two instances fully overlap (namely, large instance completely covers the small one). Therefore, the co-occurrences of instances with similar spatiotemporal characteristics are favored. We developed the $J^{*}$ measure such that it only considers relevant segments, which are the appearences of co-occurrences, and filters the rest. By filtering the nonessential parts, we aimed to develop a more appropriate and more efficient significance measure.

\subsection{Outline}

The rest of this paper is organized as follows. We will present the basic concepts and definitions related to $J^{*}$ measure in Section 2. We will discuss the antimononicity of $J^{*}$ measure (See Section 2.4). In Section 3, we will present our experiments regarding the performance of the $\mathrm{J}^{*}$ measure. Lastly, we will present conclusions in Section 4.

\section{DEVELOPING THE J* MEASURE}

\subsection{Preliminaries}

The Jaccard similarity coefficient has been extensively used for measuring the similarity and diversity among item types in shopping basket analysis [11], documents in text mining [4], or spatiotemporal instances [1]. The Jaccard similarity coefficient ( Jaccard) is calculated as follows:

$$
\operatorname{Jaccard}\left(I_{1}, I_{2}, \ldots, I_{n}\right)=\frac{\left|I_{1} \cap I_{2} \cap \ldots \cap I_{n}\right|}{\left|I_{1} \cup I_{2} \cup \ldots \cup I_{n}\right|}
$$

where item types are denoted as $I_{i}$, the numerator is the size of intersection, and the denominator is the size of the union set for given item types.

In STCOP mining [1], the spatiotemporal version of Jaccard similarity coefficient, which is denoted as $J$, is calculated as the ratio of intersection and union volumes of instances (denoted as $\left.i n s_{i}\right)$ :

$$
J\left(i n s_{1}, \ldots, i n s_{n}\right)=\frac{V\left(i n s_{1} \cap \ldots \cap i n s_{n}\right)}{V\left(i n s_{1} \cup \ldots \cup i n s_{n}\right)}
$$

where $V$ function returns the volume of trajectories by adding consecutive volumes for each timestamp. Volume for an interval is found by multiplication of corresponding area values $\left(\right.$ Area $\left._{\tau}\right)$ by time difference $\left(\Delta t=\tau_{k+1}-\tau_{k}\right)$

$$
V\left(\text { ins }_{i}\right)=\sum_{\tau=t_{\text {start }}}^{t_{\text {end }}}\left(\Delta t \times \operatorname{Area}_{\tau}\left(\text { ins }_{i}\right)\right)
$$

\footnotetext{
${ }^{1}$ Given a cce threshold, antimonotonicity for a spatiotemporal co-occurrence guarantees that if the co-occurrence is significant, then all the co-occurrences appearing among the subsets of the co-occurrence are also significant. Similarly, given a $p i$ threshold, antimonotonicity for an STCOP enforces that if the STCOP is prevalent, then all the subsets of the STCOP are also prevalent.
}

\subsection{Basic Concepts}

In this part, we will explain the basic concepts required for the calculation of the $J^{*}$ measure. As mentioned earlier, the $J^{*}$ considers only portions of the instances when calculating strength of co-occurrences.

Definition 1. Time interval list $(t i l)$ is a list of ordered time intervals. Each time interval is defined by a pair of timestamp values $\left(t_{i}, t_{j}\right)$, where $t_{i}<t_{j}$; for each $i, j ; 1 \leq$ $i<j \leq n$

$$
t i l=\left\{\left(t_{1}, t_{2}\right),\left(t_{3}, t_{4}\right), \ldots,\left(t_{n-1}, t_{n}\right)\right\}
$$

Definition 2. Interval volume function, $V_{t i l}$, calculates the volume of given trajectory-based geometries only for the time intervals given in a time interval list, denoted as til.

For a particular trajectory-based instance $i n s_{i}$, interval volume function is calculated using $V_{t i l}$ (Eq. 5). It calculates the volume of the trajectory-based instance only for those intervals specified in the time interval list $(t i l)$.

$$
V_{t i l}\left(i n s_{i}\right)=\sum_{\left[\tau_{k}, \tau_{k+1}\right) \in t i l}\left(\left(\tau_{k+1}-\tau_{k}\right) \times A r e a_{\tau_{k}}\left(i n s_{i}\right)\right)
$$

Definition 3. Co-occurrence time interval list, $t i l^{c o}$, is a time interval list calculated for two or more instances. Cooccurrence time interval list includes time intervals where there exists a spatiotemporal overlap among all the instances participating in the spatiotemporal co-occurrence.

Definition 4. In a spatiotemporal co-occurrence of instances, $P=\left\{i n s_{1}, \ldots, i n s_{n}\right\}$, the set of cross co-occurrences (denoted as xco in Eq. 6) are the events of spatiotemporal overlaps appearing among any 2 -subset of participating instances. In other words, the cross co-occurrences for $P$ are the union of pairwise co-occurrences among the instances in $P$. Given $S P \subseteq P$ and $|S P|>1$,

$$
x c o=\bigcup_{S P \subseteq P} \text { isOverlapping }(S P)=\text { true. }
$$

Definition 5. Cross co-occurrence time interval list, denoted as $t i l^{x c o}$, is a time interval list calculated for a set of spatiotemporal instances forming a spatiotemporal co-occurrence. Cross co-occurrence time interval list includes time intervals where there exists a cross co-occurrence among the subsets of participating instances.

\section{$2.3 \mathrm{~J} *$ Measure}

Let $J^{*}$ be an extended version of the spatiotemporal $J$ measure. We define the $J^{*}$ measure using the interval volume function (see Def. 2) and the cross co-occurrence time interval list (see Def. 5) as:

$$
J^{*}\left(i n s_{1}, \ldots, i n s_{n}\right)=\frac{V_{t i l^{x c o}}\left(i n s_{1} \cap \ldots \cap i n s_{n}\right)}{V_{t i l^{x c o}}\left(i n s_{1} \cup \ldots \cup i n s_{n}\right)}
$$

The $J^{*}$ measure, unlike the $J$ measure, does not particularly favor the instances who are carrying similar volume characteristics. It acknowledges the spatiotemporal cooccurrence of all participating instances as the main event of interest, while also considering the cross co-occurrences appearing among the subsets of participating instances. Regardless of unbalanced characteristics instances may have, the regions of interest for $J^{*}$ measure is only limited to cooccurrence and cross co-occurrences. Therefore, the $J^{*}$ is 
less biased when handling instances with unbalanced spatiotemporal characteristics.

Another important aspect of the problem is the storage requirements and computational complexity. From a practical point of view, it should be clear that storing only cross cooccurrences can greatly reduce storage requirements. The geometric calculations for determining unions and intersections are typically very expensive operations. Theoretically, the worst-case time complexity and storage requirements are same for $J$ and $J^{*}$. However, processing large amounts of data (mainly, checking whether there exists a spatiotemporal intersection) can take more time for $J$, as the $J^{*}$ only operates on cross co-occurrence time intervals. Hence, $J^{*}$ is more efficient measure than $J$.

\subsection{Antimonotonic Property}

Antimonotonicity is the fundemental aspect of many objective measures used in frequent pattern mining. Similar to classical frequent pattern mining approaches, in STCOP mining the antimonotonicity plays a significant role for efficiently and correctly mining the co-occurrences.

Lemma 1. $J^{*}$ is an antimonotonic measure.

Proof. Let $S$ be the set of participating instances of a spatiotemporal co-occurrence $\left(S=\left\{i n s_{1}, \ldots, i n s_{n}\right\}\right)$. Let in $s_{n+1}$ be another instance that forms a co-occurrence with all the instances in $S$. Then $S^{\prime}=\left\{i n s_{1}, \ldots, i n s_{n}, i n s_{n+1}\right\}$ and $S \subset S^{\prime}$. Then, $J^{*}(S) \geq J^{*}\left(S^{\prime}\right)$, because:

1. (1) $V_{t i l^{x c o}}\left(i n s_{1} \cap \ldots \cap i n s_{n} \cap i n s_{n+1}\right) \leq V_{t i l^{x c o}}\left(i n s_{1} \cap\right.$ $\left.\ldots \cap i n s_{n}\right)$. The intersection volume can only decrease or stay same with the addition of a new instance to the participating instance set.

2. (2) $V_{t i l^{x c o}}\left(\right.$ ins $\left._{1} \cup \ldots \cup i n s_{n} \cup i n s_{n+1}\right) \geq V_{t i l^{x c o}}\left(i n s_{1} \cup\right.$ $\left.\ldots \cup i n s_{n}\right)$. The union volume can only increase or stay same with the addition of a new instance to the participating instance set. The cross co-occurrence time interval list of $S^{\prime}$ includes at least the cross co-occurrence time interval list of $S$; and, it can potentially include cross co-occurrences between ins $_{n+1}$ and the instances in $S\left(S . t i l^{x c o} \subseteq S^{\prime} . t i l^{x c o}\right)$. Therefore, the union volume for $S^{\prime}$ is greater than or equal to the union volume of $S$.

The $J^{*}$ value for a co-occurrence decreases or stays same with the addition of a new spatiotemporal instance, as the intersection volume can only decrease or stay same and the union volume can only incraese or stay same. Hence, $J^{*}$ is an antimonotonic measure.

\section{EXPERIMENTS}

We have conducted experiments using three solar datasets to show the effects of the $J^{*}$ measure. Spatiotemporal cooccurrence pattern mining algorithm, STCOP Miner [1], is used for the experiments. We created two versions of the algorithm, with $J$ and $J^{*}$ measures. The experiments were performed in Amazon Web Services platform using one computing instance, which is listed as 'm3.xlarge', and has 15 GB of main memory and Intel Xeon-2670 processor. Three datasets used in the experiments include instances of six solar event types covering one, two, and three months of time (named as $1 \mathrm{Mo}, 2 \mathrm{Mo}$, and $3 \mathrm{Mo}$ respectively). Implementa-

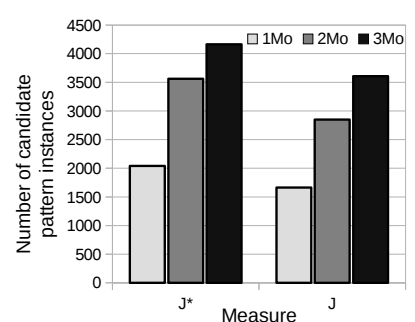

(a)

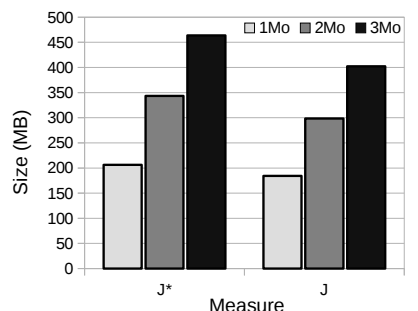

(b)
Figure 1: (a) number of generated candidate pattern instances with $J$ and $J^{*}$, (b) the total size of all geometry objects inserted to database

tion details and more information about the datasets can be found in our project website ${ }^{2}$.

\subsection{Effect of J* on Space Requirements}

The number of candidate pattern instances generated is an important indicator for space requirements, as it effects the memory usage and database size. The candidate pattern instances are the co-occurrences of at least two event instances, that may or may not pass the specified co-occurrence coefficient threshold. It can be seen in Fig. 1.a that the number of candidate pattern instances generated are similar for $J$ and $J^{*}$; yet, $J$ measure eliminates a greater portions of candidates compared to $J^{*}$. In Table 1 , we can see the percentages of registered (therefore, significant) candidate pattern instances change between $4-5.2 \%$ for $J$; and, 33-41\% for $J^{*}$. Namely, $J$ measure assesses much fewer co-occurrences as significant than $J^{*}$. However, the total sizes of all geometries inserted to database for $J$ and $J^{*}$ measures are not much different. This is because of the pruning power of the $J^{*}$ measure (only intervals of cross co-occurrences are considered for $J^{*}$, while $J$ measure obliges all geometries to be stored in the mining process). The average storage requirement of registered pattern instances in database can be seen in Table 1 . For $J$, the average size of a pattern instance varies between $1.99-2.79 \mathrm{MB}$ in three datasets, while for $J^{*}$, it varies between $0.23-0.3 \mathrm{MB}$.

Table 1: Percentage and number of registered candidate pattern instances using $J$ and $J^{*}$ measures, with the average storage requirement for a registered pattern instance

\begin{tabular}{ccccccc}
\hline \multirow{3}{*}{ Dataset } & \multicolumn{4}{c}{ Candidate Pattern Instances } \\
\cline { 2 - 7 } & $\begin{array}{c}\text { Percentage of } \\
\text { Registered }\end{array}$ & \multicolumn{2}{c}{$\begin{array}{c}\text { Number of } \\
\text { Registered }\end{array}$} & \multicolumn{2}{c}{$\begin{array}{c}\text { Average } \\
\text { size (in KB) }\end{array}$} \\
\cline { 2 - 7 } & $J$ & $J^{*}$ & $J$ & $J^{*}$ & $J$ & $J^{*}$ \\
\hline 1 Mo & 4.1 & 33.7 & 78 & 689 & 2358 & 299 \\
2 Mo & 5.2 & 41.0 & 150 & 1462 & 1990 & 234 \\
3 Mo & 4.0 & 37.4 & 144 & 1559 & 2791 & 297 \\
\hline
\end{tabular}

\subsection{Effect of $\mathrm{J}^{*}$ on Time Requirements}

Lastly, we analyze the effect of $J^{*}$ on the total time spent for geometric calculations. Geometric calculations include checking for a spatiotemporal overlap for determining the existence of a co-occurrence; and calculating the $J$ or $J^{*}$ values for each candidate pattern instance. In Fig. 2, the total time

\footnotetext{
${ }^{2}$ http://www.cs.gsu.edu/baydin2/proj/jstar.html
} 
Table 2: Average geometric calculation time for a pattern instance (in milliseconds)

\begin{tabular}{cccc}
\hline Measure & $1 \mathrm{Mo}$ & $2 \mathrm{Mo}$ & $3 \mathrm{Mo}$ \\
\hline$J^{*}$ & 16.7 & 14.4 & 14.1 \\
$J$ & 21.0 & 18.4 & 20.8 \\
\hline
\end{tabular}

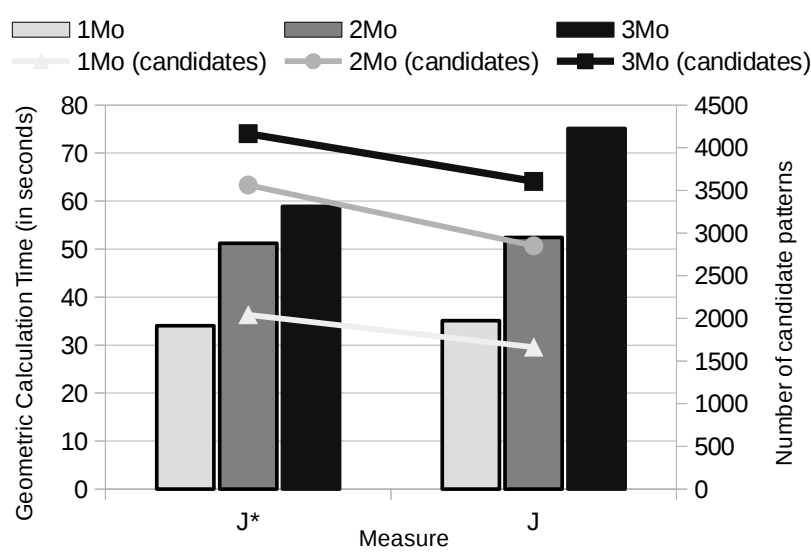

Figure 2: The total time spent on geometric calculations with $J$ and $J^{*}$. Lines show the number of candidates.

required for geometric calculations is demonstrated with the number of candidate pattern instances in minor Y-axis. The time spent on geometric calculations for both measures are similar; however, $J^{*}$ requires less time on geometric calculations, as it only considers the cross co-occurrence time intervals. The average geometric calculation time for a candidate pattern instance for $J^{*}$ is less compared to $J$, and they can be seen in Table 2. We can conclude that, for geometric operations, $J^{*}$ performs $20-33 \%$ better than $J$ on average.

\section{CONCLUSION}

In this paper, we have introduced a new significance measure, $J^{*}$, specifically designed for mining STCOPs. The $J^{*}$ is an extension to the spatiotemporal $J$ measure. We have provided the proof of antimonotonicity of $J^{*}$. For demonstrating the effects of using $J^{*}$ for assessing the strength of co-occurrences, we have conducted experiments with three solar datasets. We have experimentally demonstrated that $J^{*}$ measure can be used for efficiently mining novel STCOPs and co-occurrences. Our results also exhibit that $J^{*}$ is timeand space-efficient when compared to $J$ measure.

\section{ACKNOWLEDGMENTS}

This work was supported in part by two NASA Grant Awards (No. NNX11AM13A, and No. NNX15AF39G), and one NSF Grant Award (No. ACI1443061).

\section{REFERENCES}

[1] B. Aydin, D. Kempton, V. Akkineni, S. R. Gopavaram, K. G. Pillai, and R. A. Angryk. Spatiotemporal indexing techniques for efficiently mining spatiotemporal co-occurrence patterns. In 2014 IEEE Int. Conf. on Big Data, Big Data 2014, Washington, DC, USA, October 27-30, 2014, pages 1-10, 2014.
[2] M. Celik, S. Shekhar, J. P. Rogers, J. A. Shine, and J. S. Yoo. Mixed-drove spatio-temporal co-occurence pattern mining: A summary of results. In Proc. of the 6th IEEE Int. Conf. on Data Mining (ICDM 2006), 18-22 December 2006, Hong Kong, China, pages 119-128, 2006.

[3] L. Geng and H. J. Hamilton. Interestingness measures for data mining: A survey. ACM Comput. Surv., 38(3), 2006.

[4] M. S. Hossain and R. A. Angryk. Gdclust: A graph-based document clustering technique. In Workshops Proc. of the 7th IEEE Int. Conf. on Data Mining (ICDM 2007), October 28-31, 2007, Omaha, Nebraska, USA, pages 417-422, 2007.

[5] Y. Huang, S. Shekhar, and H. Xiong. Discovering colocation patterns from spatial data sets: A general approach. IEEE Trans. Knowl. Data Eng., 16(12):1472-1485, 2004.

[6] R. J. B. Jr. and R. Agrawal. Mining the most interesting rules. In Proc. of the 5th ACM SIGKDD Int. Conf. on Knowledge Discovery and Data Mining, San Diego, CA, USA, August 15-18, 1999, pages 145-154, 1999.

[7] Y. Morimoto. Mining frequent neighboring class sets in spatial databases. In $P$ of the 7th ACM SIGKDD Int. Conf. on Knowledge Discovery and Data Mining, San Francisco, CA, USA, August 26-29, 2001, pages 353-358, 2001.

[8] K. G. Pillai, R. A. Angryk, and B. Aydin. A filter-and-refine approach to mine spatiotemporal co-occurrences. In 21st SIGSPATIAL Int. Conf. on Advances in Geographic Information Systems, SIGSPATIAL 2013, Orlando, FL, USA, November 5-8, 2013, pages 104-113, 2013.

[9] M. Steinbach and V. Kumar. Generalizing the notion of confidence. Knowl. Inf. Syst., 12(3):279-299, 2007.

[10] P. Tan, V. Kumar, and J. Srivastava. Selecting the right interestingness measure for association patterns. In Proc. of the 8th ACM SIGKDD Int. Conf. on Knowledge Discovery and Data Mining, July 23-26, 2002, Edmonton, Alberta, Canada, pages 32-41, 2002.

[11] P. Tan, M. Steinbach, and V. Kumar. Introduction to Data Mining. Addison-Wesley, 2005.

[12] H. Xiong, S. Shekhar, Y. Huang, V. Kumar, X. Ma, and J. S. Yoo. A framework for discovering co-location patterns in data sets with extended spatial objects. In Proc. of the 4th SIAM Int. Conf. on Data Mining, Lake Buena Vista, Florida, USA, April 22-24, 2004, pages 78-89, 2004. 